\title{
A Neural Network based Model for Temperature Prediction in High Power Microwave Heating System
}

\author{
C. Chen, X.Z. Zhou, Z.Y. Tang, Y.J. Lei \\ College of Electronics and Information Engineering \\ Sichuan University \\ Chengdu, China
}

\begin{abstract}
The applications of neutral network based temperature prediction for microwave heating system has been comprehensively investigated. Temperature prediction is essential due to the characteristics of high power microwave heating system, a.k.a. big inertia and pure time-delay. This work proposes a BP neutral network based model associated with a Genetic Algorithm (GA), which aims at improving the temperature prediction accuracy. Our numerical experiments show that the BP neutral network with tansig transfer function and trainlm training function is the most appropriate one in our case based on minimum measures of error. Experiments on the pure water and coal obtained in our simulated experimental facilities were used for training and testing the proposed optimal neutral network. Based on our experimental results, the GA based BP neural network model can significantly improve the prediction accuracy as well as the convergence speed. As a result, the proposed system is suitable for the real-world applications.
\end{abstract}

Keywords-BP neural network; genetic algorithm; microwave heating; temperature prediction

\section{INTRODUCTION}

Recently, the microwave technology that is environment-friendly and high efficient shows comprehensive influence on the industrial heating technology during the new industrial revolution. Considerable efforts by the researchers are made to the study of microwave heating technology, which has already used in the practical applications of various fields. [1]The way of microwave heating is different from traditional heating method which depends on the classical principle of heat transferring. Such polarity frequently changes as the changing of the orientation of the high frequent electric field. This process provides the mutual friction between two adjacent molecules, which makes microwave generate heat [1].Consequently, compared with the traditional heating technique, the microwave heating technique is with the advantages of short reaction time, low energy consumption, and uniform heating. [2]

In this paper, a neutral network based approach has been proposed in order to improve the accuracy and efficiency of the microwave heating temperature control system. It does not require a complex mathematical model and can predict the microwave heating temperature based on the prior knowledge and online learning $[3,4]$.This proposed approach is able to be realized in the practical applications of the microwave heating systems, which has positive impact on the energy conservation and the reduction of the pollution emission.

\section{RELATED WORK AND APPROACH OVERVIEW}

\section{A. Related Works}

At present, the high-powered microwave heating has also been used in domestic enterprises, the survey found. However, the way of controlling power of microwave is also the traditional PID in enterprises.[5] Traditional linear model has limitations because of characteristics of microwave heating such as big inertia, pure time-delay and nonlinear distributed parameter.

Relevant further researches have also been done by domestic and foreign scholars. L. Momenzadeh et al. proposed a prediction model for grain microwave-assisted fluidized bed dryer based on neural network. This model used the power of microwave, drying temperature and the level of water content of grain as the input parameters of neural network, and the drying time as the prediction output of such model. Their experimental results show that their proposed model can obtain a satisfying prediction result. J. L. Pedreno-Molina et al. [6, 7] established a prediction model for microwave-assisted drying process, which is also on the basis of neural network. Sander et al. [8] investigated the dynamics analysis of the microwave based drying process for paper board. They established a BP neural network based prediction model for the level of moisture content during the drying process. Such model can also predict different drying result under different drying time and a fixed experimental condition.

It can be observed that the prediction model using neutral network for the temperature and the level of moisture content of the microwave based drying device has gained considerable attention in the last few decades. It has shown the feasibility and efficiency of the neutral network for the prediction model [9].

\section{B. Overview of the Proposed Approach}

During the last several decades, the artificial intelligence technology has already been widely applied in various areas. It brings positive change to the conventional control theory. The neural network with its unique advantage which makes it attract considerable attention.[10] The proposed approach uses genetic algorithm to optimize BP neural network in order to improve the reliability, robustness and efficiency of the BP neutral network in the case of the temperature prediction of the microwave heating system. The predicted temperature of heated materials can help adjust the control parameters and the 
output power of microwave. As a result, a real-time and efficient control of microwave heating system can be achieved.

\section{Contribution of the proposed approach:}

1. A detailed investigation of the characteristics of microwave heating is performed.

2. An intelligent control system is proposed based on the properties of high-power industrial microwave heating.

3. The introduction of GA can help avoid the BP neutral network falling into the local optimum and improve the training efficiency of the whole system.

4. The proposed approach is tested using the experimental and practical data, and the obtained results validate our arguments.

\section{PROPOSED APPROACH}

\section{A. System Overview}

In order to improve the accuracy of the temperature prediction for the microwave heating, we first build a BP neutral network, then through genetic algorithm to optimize BP neutral network. The genetic algorithm includes three operations: selection, crossover and mutation. After those operations, the BP neutral network optimized used to train.

\section{B. BP Neural Network}

BP (Back Propagation) Neural network is a variant of neural network with at least three layers, which includes the input layer, output layer and hidden layer. The training phase consists of forward and reverse transfer process, and the corresponding input information is transmitted to the output layer via the hidden layer. Then, the output is compared with the prediction in order to adjust its parameters. If significant error exists, the error is returned along the original path based on the back propagation algorithm. Accordingly, the connection weights between the input layer and the output layer (through the hidden layer) are adjusted in order to minimize the corresponding errors. After continuous adjustment, the error will be reduced gradually until it reaches a predefined accuracy [7].

\section{Genetic Algorithm}

Inspired by the natural selection and genetics, Genetic Algorithm (GA) is developed as an adaptive heuristic search algorithm based on a random search aiming to solve the optimization problems.GA exploits the previous information to conduct such search restricted within the region which can provide better performance. The fundamental idea of GA is presented to mimic the natural evolution system following the principles first proposed by Charles Darwin of 'survival of the fitness'. In nature condition, individuals compete for limited resources, and it results in the survival of fitness individuals and extinction of the weaker ones.

Compared with the conventional Artificial Intelligence (AI) systems, GA is more robust under noise and the slight change of inputs. More specifically, GA can offer significantly better search result over a large state-space, multi-modal state-space or a high-dimensional space than the older optimization techniques.

\section{EXPERIMENTAL RESULTS}

In this work, two experiments have been conducted to validate our proposed approach. In the first experiment, we use the pure water as the heated material. In the second one, we use the coal instead of pure water to simulate the real-world applications. The corresponding data are recorded at the same time. Then the obtained data is used to train the neural network model. Finally the trained neural network is used to predict the temperature during the heating process.

\section{A. Experimental Setup}

We first conduct an experiment on the pure water in order to test the performance of the proposed approach. The experimental facility consists of four components, the microwave generator, wave-guide component, microwave cavity, and system controller. The experimental parameters are set as follows: the temperature is constant to be $20{ }^{\circ} \mathrm{C}, 2$ liters water in an uncapped container, the thermometer is located 1 $\mathrm{cm}$ under the water surface. The initial temperature of water is $21{ }^{\circ} \mathrm{C}$, and final temperature for heating is $50{ }^{\circ} \mathrm{C}$.

In this subsection, we conduct another experiment based on the real-world microwave coal heating facility. The power of the magnetron microwave source is automatically controlled by our proposed temperature prediction model according to the detected instant input temperature and reflection power. Our microwave coal heating facility consists of the following modules: microwave power control system, conveyor belt, temperature sensor, microwave reflection power sensor.

\section{B. The Proposed Prediction Model}

In the proposed approach, the number of input layer and output layer are manually determined. However, the number of hidden layer only can be determined according to the former experience as the following formula:

$$
f=\sqrt{m+n}+a
$$

where $m$ and $n$ represent the cell number of input layer and output layer respectively. Additionally, $a$ is a constant ranging between 0 and 10 . Therefore, we select the cell number of hidden layer between 3 and 15 in this proposed approach.

During the training process, the cell number of hidden layer can be determined when the MSE of the learned neutral network reaches the minimum by changing the number from 3 to 15 .

In this paper, tansig and purelin are selected as the transfer function of input layer and output layer respectively. trainlm is selected as the training function. The data was collected at an interval of 2 second. Then one fifth of collected data is selected uniformly to form the final training samples, which results in 107 sets of training samples. This proposed approach applied the neutral network toolbox of MATLAB, and the parameters are set as followings:

Parameters-setting as follows:

Max Training Time: net.trainParam.epochs $=100$

Training Goal: net.trainParam.goal=0.0004 
Learning Rate: net.trainParam.lr=0.1

80 sets of data were selected as to the predicting samples.

The predicted values of the conventional BP neural network and the actual value are shown in Fig.1. The convergence of the algorithm is shown in Fig.2. The errors of the BP neural network algorithm are shown in Fig.3. The average relative error was 0.0020 .

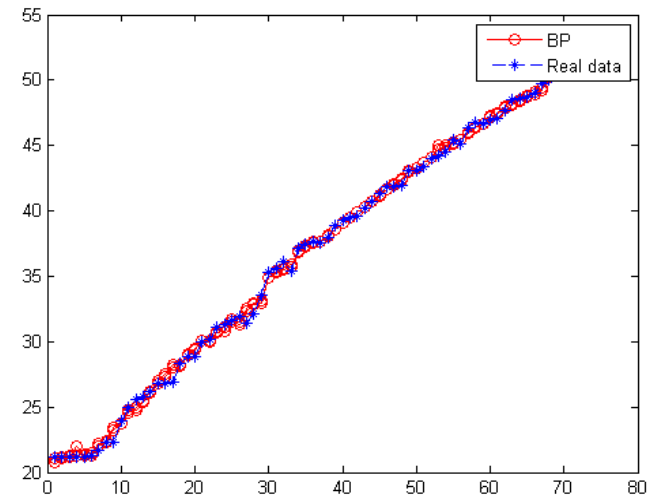

FIGURE I. COMPARISON OF THE ACTUAL AN THE PREDICTED TEMPERATURE BY THE BP NEURAL NETWORK

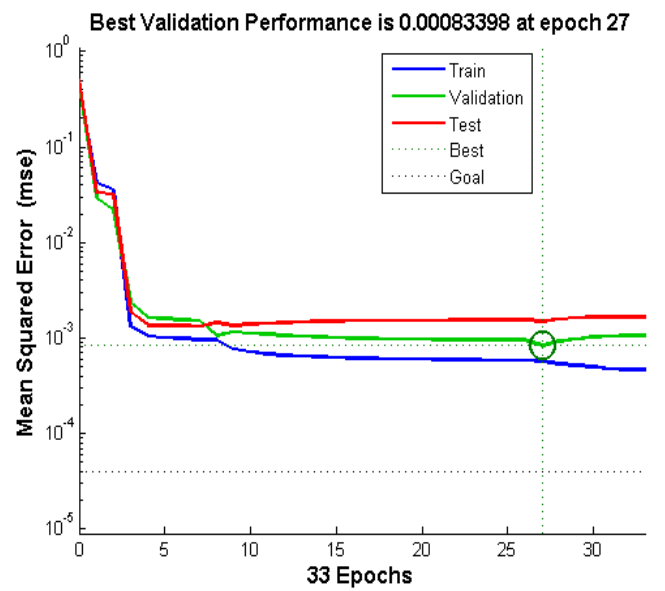

FIGURE II. CONVERGENCE OF THE BP NETWORK

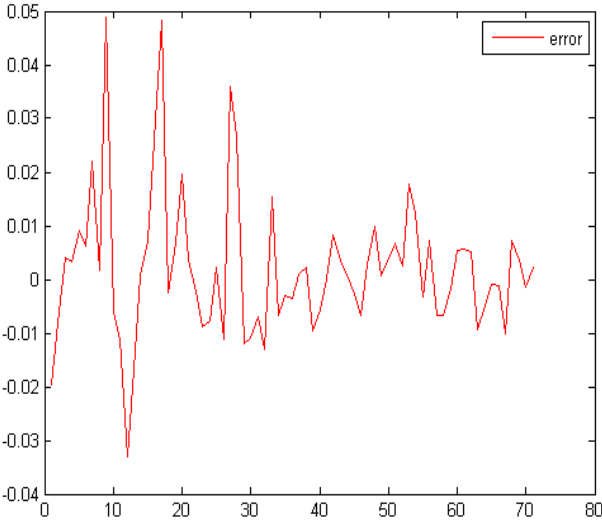

FIGURE III. $\quad$ ERRORS OF THE BP NETWORK

In the industrial heating coal process, the prediction model adopted in this paper was the same as the model of heating pure water. BP neural network was also selected to predict temperature. The coal would be heated from the initial temperature to the maximum temperature $150{ }^{\circ} \mathrm{C}$. During the process of heating system, the temperature of coal was collected and recorded every 30 seconds, at the same time, the input power and the reflected power were also collected and recorded. The predicted values of the BP neural network and the actual value are shown in Fig.4. Convergence of the algorithm is shown in Fig.5. The error of the BP neural network algorithm is shown in Fig.6. The average relative error was 0.0035 .

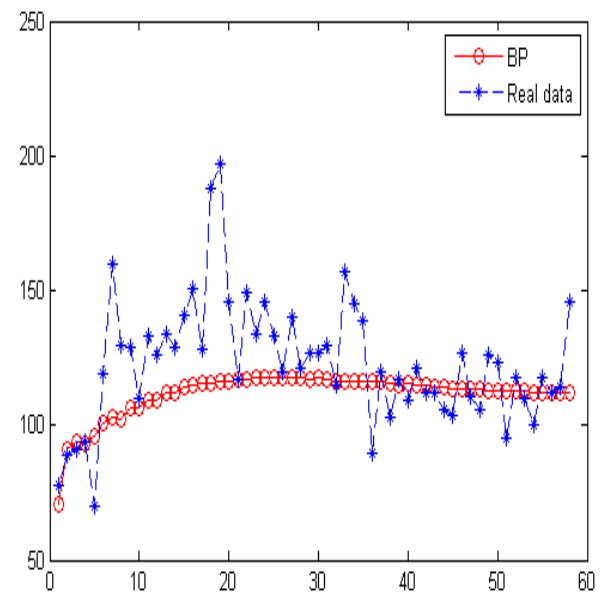

FIGURE IV. COMPARISON OF THE ACTUAL AND THE PREDICTED TEMPERATURE BY THE BP NETWORK 


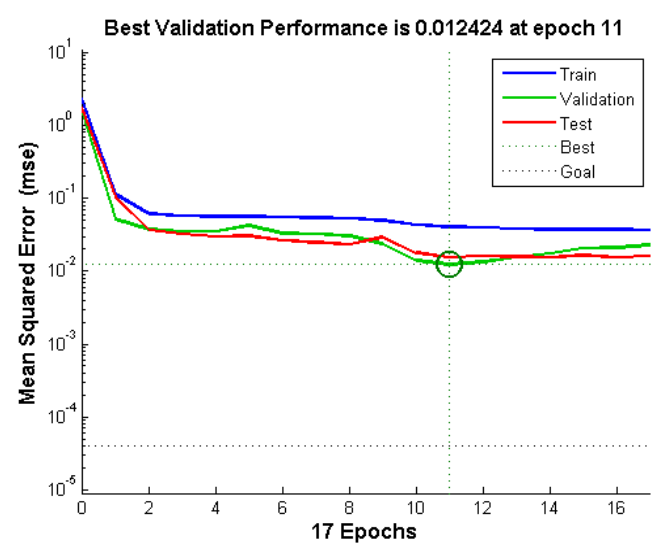

FIGURE V. CONVERGENCE OF THE BP NETWORK

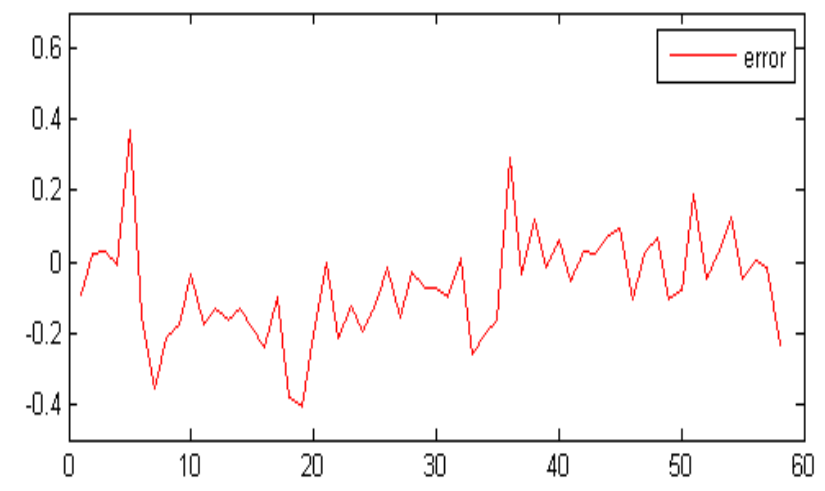

FIGURE VI. ERRORS OF THE BP NETWORK

\section{Using GA to Optimize the BP Neural Network}

In this proposed approach, Crossover probability of the GA is set to 0.2 and mutation probability is set to 0.1. After continuous learning, 30 is determined as the the size of the new population. With this model, the initial weights and thresholds of the neural network have been optimized by GA to train the neutral network based prediction model.

The predicted values of the GA-BP neural network and the actual values are shown in Fig.7. The convergence of the algorithm is shown in Fig.8. The errors of the GA-BP neural network algorithm are shown in Fig.9. The average relative error was 5.6716e-04.

In the industrial heating coal process, the prediction model adopted in this paper was the same as the model of heating pure water. The coal would be heated from the initial temperature to the maximum temperature $150{ }^{\circ} \mathrm{C}$.

The prediction by the GA-BP neural network and the actual value is shown in Fig.10. Convergence of the algorithm is shown in Fig.11. The errors of the GA-BP neural network algorithm are shown in Fig.12. The average relative error was 0.0035 .

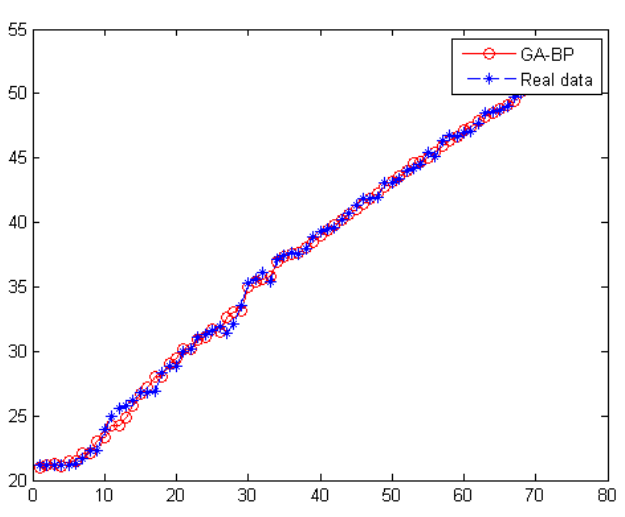

FIGURE VII. COMPARISON OF THE ACTUAL AND THE PREDICTED TEMPERATURE BY THE GA-BP NETWORK

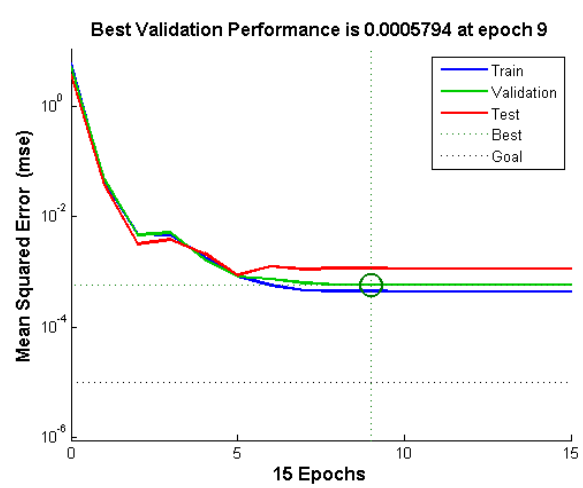

FIGURE VIII. CONVERGENCE OF THE GA-BP NETWORK

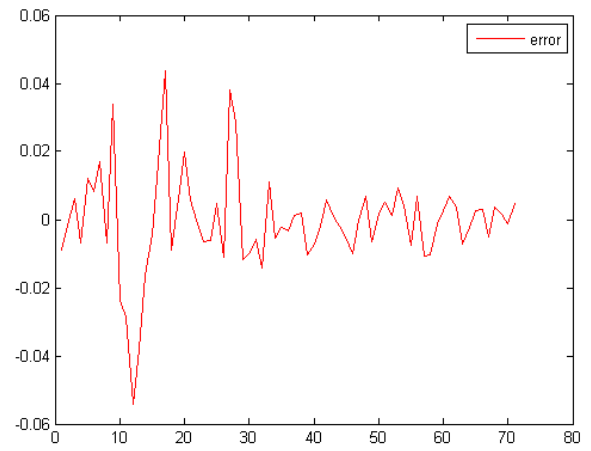

FIGURE IX. IRRORS OF THE GA- BP NETWORK 


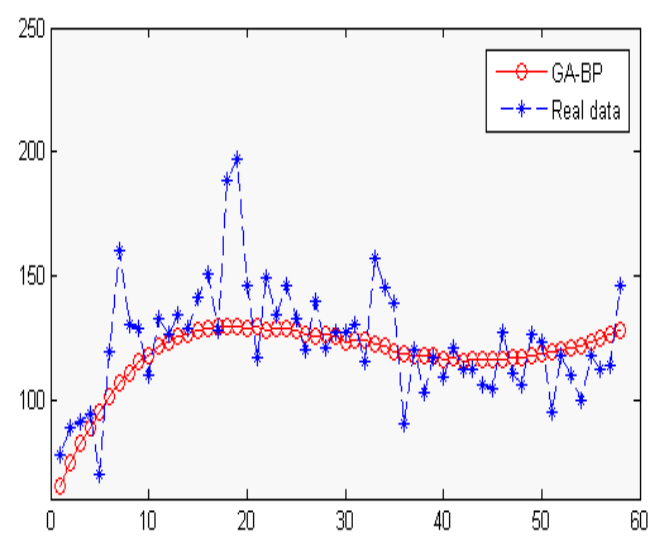

FIGURE X. COMPARISON OF THE ACTUAL AND THE PREDICTED TEMPERATURE BY THE GA-BP NEURAL NETWORK

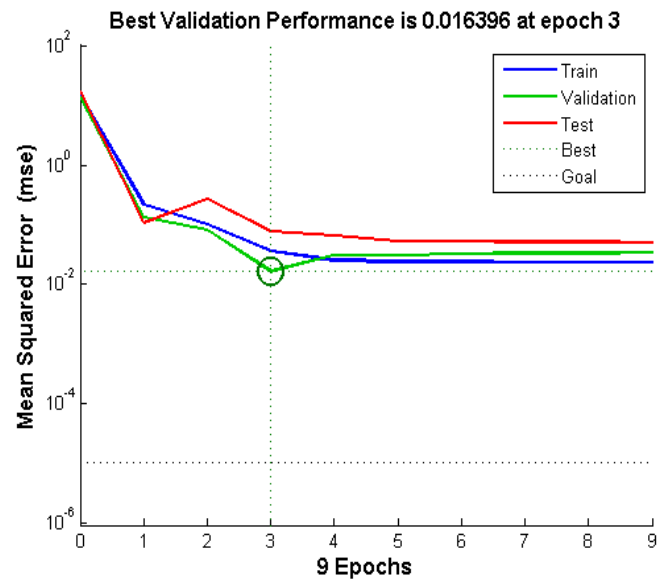

FIGURE XI. CONVERGENCE OF THE GA-BP NETWORK

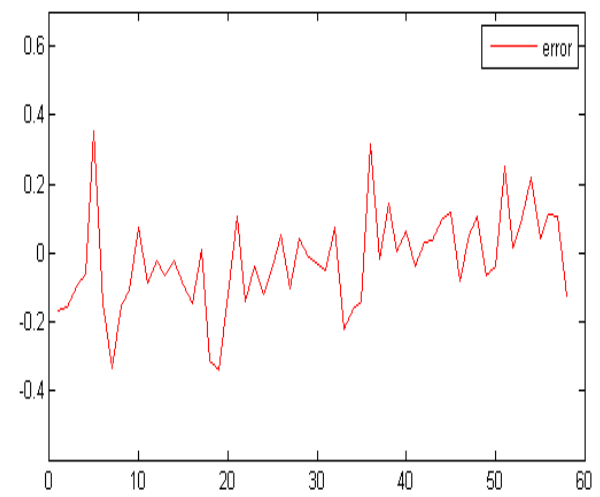

FIGURE XII. ERRORS OF THE GA- BP NETWORK

D. The Comparison of the Predicted Values of the BP Neural Network and the GA-BP Neural Network

In the first experiment, 80 sets of data are selected for test. The first and last 10 sets of the predicated data (20 in total) are compared with their actual data in order to calculate the relative error and the results are listed in Table1 and Table2.
TABLE I. THE FIRST TEN SETS OF DATA

\begin{tabular}{lcl|ccc}
\hline & BP & & \multicolumn{3}{|c}{ GA-BP } \\
\hline Prediction & Actual & $\begin{array}{l}\text { Relative } \\
\text { Error }\end{array}$ & Prediction & Actual & $\begin{array}{l}\text { Relative } \\
\text { Error }\end{array}$ \\
20.7865 & 21.2 & -0.0195047 & 21.0128 & 21.2 & -0.00883 \\
21.0324 & 21.2 & -0.0079057 & 21.1692 & 21.2 & -0.00145 \\
21.2883 & 21.2 & 0.00416509 & 21.3265 & 21.2 & 0.005967 \\
21.2714 & 21.2 & 0.00336792 & 21.0533 & 21.2 & -0.00692 \\
21.3944 & 21.2 & 0.00916981 & 21.4574 & 21.2 & 0.012142 \\
21.4332 & 21.3 & 0.00625352 & 21.4741 & 21.3 & 0.008174 \\
22.1744 & 21.7 & 0.02186175 & 22.0662 & 21.7 & 0.016876 \\
22.3376 & 22.3 & 0.0016861 & 22.1439 & 22.3 & -0.007 \\
23.3881 & 22.3 & 0.04879372 & 23.0576 & 22.3 & 0.033973 \\
23.7519 & 23.9 & -0.0061967 & 23.3335 & 23.9 & -0.0237 \\
Average Relative Error: & 0.0128905 & Average & Relative & Error \\
\hline
\end{tabular}

TABLE II. THE LAST TEN SETS OF DATA

\begin{tabular}{|c|c|c|c|c|c|}
\hline \multicolumn{3}{|c|}{$\mathrm{BP}$} & \multicolumn{3}{|c|}{ GA-BP } \\
\hline $\begin{array}{l}\text { Predicti } \\
\text { on }\end{array}$ & Actual & $\begin{array}{l}\text { Relative } \\
\text { Error }\end{array}$ & $\begin{array}{l}\text { Predicti } \\
\text { on }\end{array}$ & Actual & $\begin{array}{l}\text { Relative } \\
\text { Error }\end{array}$ \\
\hline 47.9414 & 47.7 & 0.0050608 & 47.8819 & 47.7 & 0.003813 \\
\hline 48.0491 & 48.5 & -0.0092969 & 48.1522 & 48.5 & -0.00717 \\
\hline 48.3644 & 48.6 & -0.0048477 & 48.4963 & 48.6 & -0.00213 \\
\hline 48.6614 & 48.7 & -0.0007926 & 48.8263 & 48.7 & 0.002593 \\
\hline 48.94 & 49 & -0.0012245 & 49.1419 & 49 & 0.002896 \\
\hline 49.2004 & 49.7 & -0.0100523 & 49.4432 & 49.7 & -0.00517 \\
\hline 50.3575 & 50 & 0.00715 & 50.1774 & 50 & 0.003548 \\
\hline 50.5748 & 50.4 & 0.00346825 & 50.4778 & 50.4 & 0.001544 \\
\hline 50.8274 & 50.9 & -0.0014263 & 50.8351 & 50.9 & -0.00128 \\
\hline 51.1109 & 51 & 0.00217451 & 51.2487 & 51 & 0.004876 \\
\hline \multicolumn{3}{|c|}{$\begin{array}{c}\text { Average Relative Error: } \\
0.00454939\end{array}$} & \multicolumn{3}{|c|}{$\begin{array}{c}\text { Average Relative Error: } \\
0.003501782 \\
\end{array}$} \\
\hline
\end{tabular}

\section{E. Conclusion Analysis}

Four conclusions can be drawn from the corresponding experiments:

(1) The results of BP model and GA-BP model are both acceptable. First, the number of training samples is sufficient for generating a high-quality prediction model. Second, sampling interval is short which guarantees that the change of the temperature between each time interval is minor.

(2) GA-BP model is able to overcome the inherent disadvantage of the conventional $\mathrm{BP}$ neural network. The reason is that the initial weights and thresholds of the neural network are optimized using GA to train the neural network so that it could provide higher prediction accuracy.

(3) Our experimental results show that in the first experiment, the BP neural network convergence requires 27 steps while GA-BP neural network convergence requires only 9 steps. In the industrial heating coal process, the BP neural network convergence requires 11 steps while GA-BP neural network convergence requires only 3 steps. As a result, the time efficiency of the GA-BP network is significantly better than the conventional BP neutral network. 
(4) In real-world applications (the microwave coal heating experiment), the proposed approach is disturbed by the environmental factors, which makes the prediction accuracy of the corresponding experiment decrease significantly.

\section{SUMMARY}

In this paper, a GA-BP neural network model has been proposed which can be applied to automatically control the microwave power in the high-power industrial heating systems. We conducted a number of experiments in order to validate our proposed approach. The experimental results show that the genetic algorithm based BP neutral network has a better computational efficiency and high prediction accuracy when compared with the conventional BP neutral network. We believe that our proposed approach can be subject to improvements. Our work mainly depends on the thermal sensors for real-time temperature detection, which may cause the outlier points and unexpected noises.

In our future work, we will propose a more precise algorithm to compensate the error caused by the sensors and we believe it will result in a more precise prediction results.

\section{ACKNOWLEDGEMENTS}

This work was supported by a grant from the National Basic Research Program of China (973 Program) (No. 2013CB328903)

\section{REFERENCES}

[1] J.M. Woo Seong Che, The Study on the Uniform Drying of Microwave Heating System with Multiple Waveguides, Applied Mechanics and Materials, pp. 219-223, 2013.

[2] P. Rattanadecho, The simulation of microwave heating of wood using a rectangular wave guide: Influence of frequency and sample size, Chemical Engineering Science, pp. 4798-4811, 2006.

[3] S. Haykin, Neural Networks and Learning Machines, Prentice Hall, 2008.

[4] N. Costa, and S. Haykin, Multiple-Input, Multiple-Output Channel Models: Theory and Practice, Wiley-Interscience, 2010.

[5] J. Li, H. Feng, and S. Li, Wavelet prediction fuzzy Neural Network of the annealing furnace temperature control, Book Wavelet prediction fuzzy Neural Network of the annealing furnace temperature control, Series Wavelet prediction fuzzy Neural Network of the annealing furnace temperature control,ed., Editor ed. IEEE, pp. 940-943, 2011.

[6] J.L. Pedreño-Molina, J. Monzó-Cabrera, A. Toledo-Moreo, and D. Sánchez-Hernández, A novel predictive architecture for microwave-assisted drying processes based on neural networks, International Communications In Heat And Mass Transfer, pp. 1026-1033, 2005.

[7] J.L. Pedreño-Molina, J. Monzó-Cabrera, and D. Sánchez-Hernández, A new predictive neural architecture for solving temperature inverse problems in microwave-assisted drying processes, Neurocomputin, pp. 521-528, g2005.

[8] A. Sander, N. Bolf, and J.P. Kardum, Research on dynamics and drying time in microwave paper drying, Chemical And Biochemical Engineering Quarterly, pp. 159-164, 2003.

[9] P.P. Tripathy, and S. Kumar, Neural network approach for food temperature prediction during solar drying, International Journal Of Thermal Sciences, pp. 1452-1459, 2009.

[10] H. Zhou, L. Zheng, J. Fan, and K. Cen, Application of general regression neural network in prediction of coal ash fusion temperature, Journal of Zhejiang University, pp. 1479-1482, 2004. 